OPEN

SUBJECT AREAS: THEORETICAL ECOLOGY

PALAEOECOLOGY

Received

12 August 2014

Accepted

24 December 2014

Published

29 January 2015

Correspondence and requests for materials should be addressed to K.Y. (kyoshida@nies. go.jp)

\title{
Properties of ecosystems that are vulnerable during eco-fusion
}

\author{
Katsuhiko Yoshida' \& Kei Tokita ${ }^{2}$
}

${ }^{1}$ Center for Environmental Biology and Ecosystem Studies, National Institute for Environmental Studies, 16-2 Onogawa, Tsukuba, Ibaraki 305-8506, Japan, ${ }^{2}$ Department of Complex Systems Science, Graduate School of Information Science, Nagoya University, Furo-cho, Chikusa-ku, Nagoya, Aichi 464-8601, Japan.

When two ecosystems with separate evolutionary histories come into contact (eco-fusion), reciprocal invasions occur during their fusion. Asymmetries in the migration direction or extinction rate then occur (e.g., during the Great American Biotic Interchange, GABI). Hypotheses have been proposed to describe this process, but the ecosystem properties have not been adequately discussed. To identify the ecosystem properties that create vulnerability to species loss during eco-fusion, we conducted computer simulations of the fusion of ecosystems with independent evolutionary histories. With asymmetrical species extinction rates, the ecosystem with a higher extinction rate had a shorter food chain, a higher ratio of animal species to plant species, and a lower ratio of carnivores to herbivores. Most ecosystems that have undergone isolated evolution are vulnerable. These results may explain the vulnerability of South America's ecosystem during the GABI and that of modern Australia.

W hen geographical barriers disappear (e.g., due to continental drift or climate change), ecosystems with long separate evolutionary histories come into contact and reciprocal invasions occur ("eco-fusion"). Such events have occurred frequently in Earth's history. During these events, asymmetries in the direction of migration or in the magnitude of extinction have been frequently observed ${ }^{1}$. The Great American Biotic Interchange (GABI) is a famous example ${ }^{2}$. Several hypotheses have been proposed to explain the asymmetries: passive transport by unidirectional currents (e.g., the Suez Canal ${ }^{3,4}$ ), marine interchange across the tropical Pacific during the Pleistocene under the effect of the North Equatorial Countercurrent ${ }^{5,6}$, niche vacancy (e.g., the Trans-Arctic Interchange ${ }^{7}$ ), the effects of climate change ${ }^{8-11}$, differences in the continental area ${ }^{12,13}$, and biological superiority (e.g., competition, defense, reproduction ${ }^{14-22}$ ). Among these hypotheses, biological and ecological factors used to be emphasized by researchers, but recently they have not been received much atten$\operatorname{tion}^{8,10,23}$. However, considering the impact of today's biological invasions, it seems highly likely that large-scale reciprocal invasions occurred as soon as geographical barriers broke down and played a key role in the resulting changes of the biota.

Previous research did not consider the fragility of an ecosystem's structure. Thus, it is not clear whether an ecosystem was vulnerable due to a fragile structure (e.g., due to its evolutionary history, environmental conditions) and whether that fragility caused the abovementioned asymmetries. To investigate this subject, comparisons are needed between the structure of "winner" and "loser" ecosystems $\mathrm{s}^{24}$. However, such comparisons have not been done because the structure of paleoecosystems (e.g., the topology of the network of interspecific interactions) is not supported by sufficiently strong empirical evidence. When empirical studies are impossible, hypothetical studies (e.g., modeling) can provide insights that clarify our understanding of the phenomena. Gilpin $^{25}$ conducted pioneering modeling work based on this concept. However, Gilpin's model was too simple to fully describe eco-fusion. In the present study, we conducted computer simulations using a more advanced ecosystem model for two ecosystems with independent evolutionary histories. Our goal was to identify the ecosystem properties that caused vulnerability during eco-fusion.

We utilized the ecosystem model of Yoshida ${ }^{26}$; details are provided in Supplementary Material 1. Briefly, the model examines ecosystems with both animal and plant species. The model therefore accounts for both autotrophs and heterotrophs, and their positions within a food chain or food web. Each species has specific characteristics. Plant species with similar characteristics compete. Animal species feed on other species that are suitable for their dietary needs and preferences. Animal species with a narrow range of needs and preference become specialists with higher assimilation rates. Population dynamics is calculated using the multidimensional Lotka-Volterra equation. In each time step, the model calculates the population dynamics for all species. If the 
total biomass of a species becomes smaller than the individual body size of the species (the biomass of one individual of the species), the species becomes extinct.

Every 100 time steps, speciation occurs for a randomly chosen species. The new species' characteristics are defined by slightly modifying those of its ancestor using a random number sampled from a Gaussian distribution. Interactions with other species are defined based on the characteristics of the new species. Every 500 time steps, an entirely new species appears (e.g., to mimic dispersal of propagules or individuals by windblown plant seeds or insects arriving at an island on floating debris) and becomes the founder of a new lineage. The arrival of new plant and animal species alternates. The new species characteristics are chosen randomly (see Supporting Material 1). Interactions with other species are defined based on the characteristics of the new species, as in the case of species that arise through speciation. In a completely isolated ecosystem, new species would never appear in this way and would only arise through speciation. Ecosystems in the model evolve via repetition of these processes.

When simulating eco-fusion, we must consider how to construct interactions between species that have different evolutionary histories. To solve this problem, it is useful to construct interspecific interactions based on species characteristics ${ }^{26}$. In Yoshida's model ${ }^{26}$, two types of interspecific interaction (between invasive and native species, and between two native species) can be constructed in the same manner regardless of the previous histories of the species. Therefore, the effect of biological invasion can be assessed accurately. In addition, this model can reflect the evolutionary history of species to interspecific interaction between them, because species characteristics are the results of evolution. We used Yoshida's parameter set ${ }^{26}$ because it reproduced the properties of real ecosystems well.

Using Yoshida's model ${ }^{26}$, two ecosystems were constructed in a single simulation. Their initial states were set in the same manner. Both ecosystems evolve independently. In this model, the ecosystem typically reaches equilibrium after 100000 time steps ${ }^{27}$. To ensure equilibrium, we used 200000 time steps before eco-fusion in the present study.

Next, the two ecosystems are connected and species migration occurs. Migration events occur every 100 time steps at a migration rate defined separately for each species. The rate represents the ratio of the biomass of the species that is able to migrate to the neighboring ecosystem and is determined using a random number uniformly distributed between 0 and 0.1 . When the biomass that is able to migrate is smaller than that of an individual of a species, the species cannot migrate at that time. The migration parameter may influence the magnitude of an invasion's impact. We conducted simulations to determine how the results of eco-fusion depend on the migration rate (Supporting Material 2) and found no significant difference in the rate of appearance of asymmetry in the survival rate or in ecosystem properties. Thus, we will present the results of simulations with the migration rate set as described in this section. Interspecific interactions among migrants are preserved in the destination. When species meet new species after eco-fusion, interspecific interactions between them are set in the same manner mentioned above. After eco-fusion, the appearance of new species ceases. Simulations are stopped at the 210 000th time step (i.e., at 10000 time steps after eco-fusion).

In this study, we simulated four types of eco-fusion. In type 1, both ecosystems evolve under the default conditions used by Yoshida ${ }^{26}$. In types 2 to 4, the control ecosystem evolves under the default conditions (relatively high and constant primary production and the appearance of entirely new species), but the experimental ecosystem evolves under the following conditions: (2) low primary production, (3) fluctuation of primary production, and (4) completely isolated (no appearance of entirely new species) during the evolution before ecofusion. Type 1 was iterated 1000 times, versus 500 times for types 2 to 4. The results presented here represent the means of those iterations.

\section{Results}

Asymmetries after eco-fusion. First, we investigated the frequency of asymmetries in survival rates of native species and the migration success rate. The survival rate equals the number of native species that survived at the end of each simulation divided by the number in the ecosystem before eco-fusion. The migration success rate equals the number of species that migrate to the recipient ecosystem divided by the total number of species in the donor ecosystem. When these rates differ between the two ecosystems by more than 10 percentage points, we defined this as asymmetrical. (For example, when the survival rate of an ecosystem is $25 \%$ and that of the other ecosystem is $10 \%$, the difference in the survival rate is 15 percentage points. In this case, an asymmetry in the survival rate is observed in the pair.) For the survival rate in the type 1 simulation, asymmetry occurred about $30 \%$ of the time (294/1000). The maximum difference in the rates between the winner and loser ecosystems was 34.7 percentage points. Here, we define a "winner" ecosystem as one that exhibited a higher survival rate than the other system in a given simulation; the other ecosystem was a "loser." For the migration success rate, we observed asymmetry in about $50 \%$ of the simulations (474/1000), and the maximum difference in the rate was 42.9 percentage points. Ecosystems with low survival rates after eco-fusion can be defined as vulnerable to eco-fusion, and those with low migration success rates can also be defined as vulnerable. We found a statistically significant relationship between the two rates (Fig. S1), indicating that an ecosystem that loses in terms of its migration success rate also loses in terms of its survival rate. Thus, in this study, we analyzed the results by focusing on ecosystems with a low survival rate.

Properties of vulnerable ecosystems. To clarify the properties of a vulnerable ecosystem (i.e., one that may become a loser), we focused on the 294 simulations with an asymmetric survival rate and compared the properties of winner and loser ecosystems before the eco-fusion (Fig. 1, Table S1). Loser ecosystems had a high ratio of animal species to plant species and a shorter food chain length. Of the animal species in loser ecosystems, the proportion of herbivores was high, whereas that of carnivores was low. The proportion of intermediate species (which feed on prey and are fed on by predators) was also low. The proportion of top species (those that have no predators) was high, and the top species directly fed on basal (plant) species (the value of $\% T-B$ was high; Fig. 1, Table S1). These results indicate that in loser ecosystems a small number of plant species supported a large number of herbivorous animals that were not fed on by predators, and the resulting food chain length was short (Fig. 2). In loser ecosystems, plants had low biomass, because they were grazed by many herbivores (Table S2). As a result of the insufficient food supply, the biomass of herbivores also became small (Table S2).

Certain changes occurred in loser ecosystems after eco-fusion. The proportion of top species $(\% T)$ decreased remarkably, and the proportions of intermediate species $(\% I)$ and basal species $(\% B)$ increased (Fig. 3, Table S3). The network structure in loser ecosystems also changed. The proportion of direct feeding of top species on basal species $(\% T-B)$ decreased remarkably, whereas the proportion of interactions between intermediate species $(\% I-I)$ and that between intermediate and basal species $(\% I-B)$ both increased remarkably (Fig. 3, Table S3). In addition, the maximum food chain length increased (Fig. 3, Table S3). These results indicate that herbivores in loser ecosystems that were not fed on by predators began to be fed on by predators that invaded from the winner ecosystem after ecofusion. As a result, many herbivores in loser ecosystems, which had small biomass (Table S2), could not resist the increased predation pressure from invaders and became extinct.

Plant (basal) species also invaded from the winner ecosystem. As a result, the number of plant species increased (Table S3) and the 


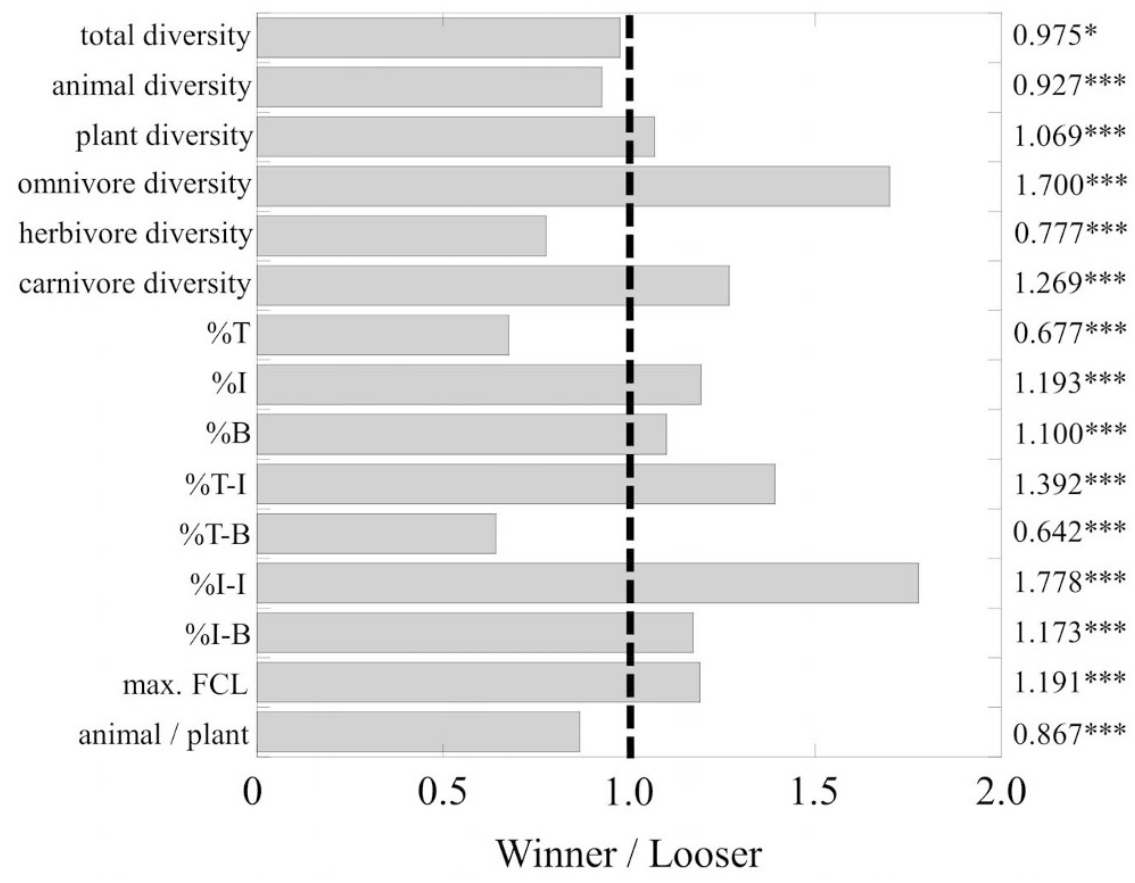

Figure 1 Comparison of the properties of winner and loser ecosystems before the eco-fusion. For each parameter, the ratio of the value for the winner ecosystem to the value for the loser ecosystem is shown. The actual ratios and their significance are shown at the right side of the diagram

(Mann-Whitney $U$-test; ${ }^{*} p<0.05,{ }^{* *} p<0.01,{ }^{* * *} p<0.001$ ). Table S1 presents details of the calculations. Abbreviations: $\% T, \% I$, and $\% B$ are the proportions of top species (without predators), intermediate species (with both predators and prey), and basal species (plants); \% $T-I, \% T-B, \% I-I$, and $\% I-B$ represent the proportions of interactions between the two types of species; max. FCL represents the maximum food chain length in an ecosystem; and animal/plant represents the ratio of animal species to plant species.

number of competitors of native plant species in loser ecosystems increased (Table S4). Because the biomass of plant species in loser ecosystems was lower than that in winner ecosystems (Table S2), plant species in the loser ecosystem were at risk of extinction. The extinction of plant species might be another cause of the loss of herbivores. Therefore, loser ecosystems exhibited high overall extinction rates.

In winner ecosystems, different processes occurred. Most plant species had large biomass (Fig. 1, 2; Table S1), so they could resist the increased competition and grazing pressure. Most herbivores also had high biomass (Table S2) and could resist the competition for resources with herbivores that migrated from the loser ecosystem. In addition, because carnivores in loser ecosystems had a smaller body size than in winner ecosystems, they could not compete with the more powerful carnivores in winner ecosystems. Therefore, in winner ecosystems, predation pressure did not increase, and this pressure did not appear to become a cause of species extinction.

Temporal changes in species diversity. Analysis of the temporal diversity patterns suggests how loser ecosystems developed. At the beginning of evolution, the plant species diversity decreased in both winner and loser ecosystems (Fig. 4a), because of the initial instability of the system. At that time, the plant species diversity in loser ecosystems was lower than that in winner ecosystems. Afterward, the plant species diversity in loser ecosystems stabilized at a lower level than that in winner ecosystems (Fig. 4a). When the plant species diversity decreased, an energy constraint developed ${ }^{28}$. Because the energy constraint was more severe at higher trophic levels in the loser ecosystems, the carnivore and omnivore species diversity increased only temporarily, and stabilized after about 40000 time steps; in contrast, it increased continuously in winner ecosystems (Fig. 4b). As a result, predation pressure on herbivores decreased in loser ecosystems, and the herbivore species diversity increased; this parameter also increased in winner ecosystems, but the number of species stabilized sooner (Fig. 4c). The high herbivore diversity created high grazing pressure on the plant species in loser ecosystems. Therefore, the plant species diversity decreased (Fig. 4a) after 100000 time steps. The decreased plant species diversity in turn created the abovementioned energy constraint, and this feedback loop made the ecosystems vulnerable; that is, (a) winner

(b) loser

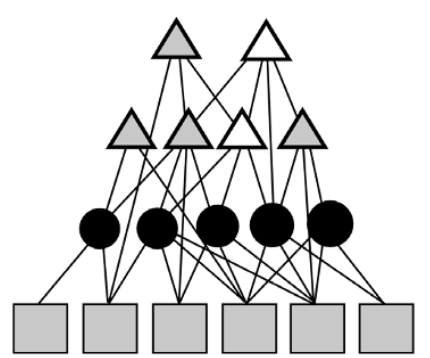

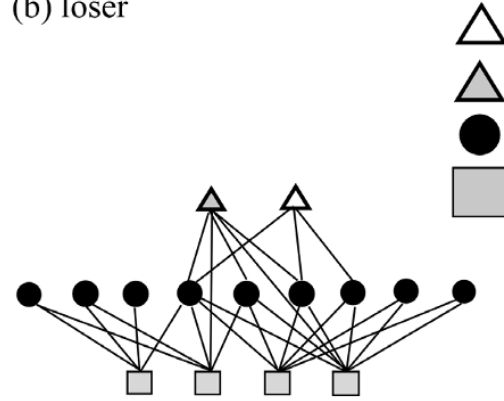

carnivore

omnivore

herbivore

plant

Figure $2 \mid$ Schematic illustrations of the food chains in (a) winner and (b) loser ecosystems. The size of each symbol schematically shows the amount of biomass of each species. 


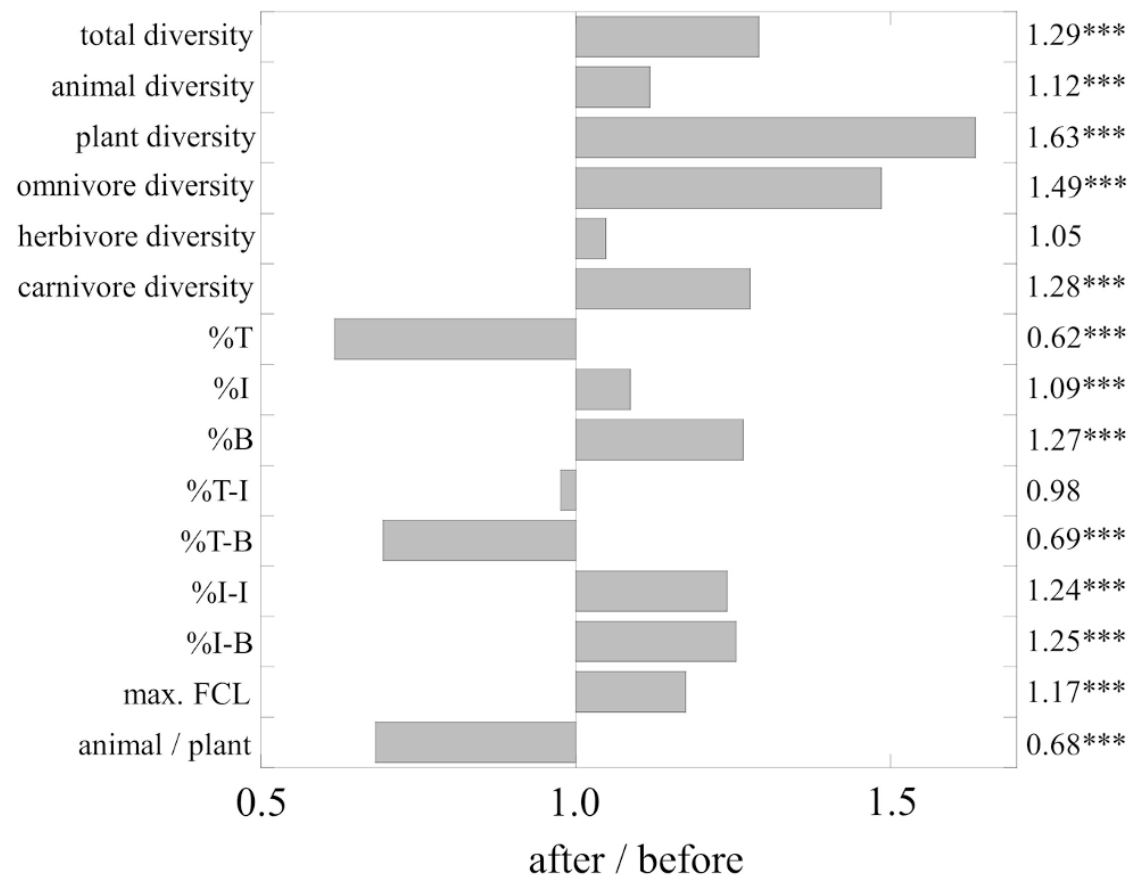

Figure $3 \mid$ Change in the properties of a loser ecosystem after eco-fusion. The horizontal axis represents the ratio of the mean value of each parameter after eco-fusion (at the end of the simulation) to the value before eco-fusion. For abbreviations, see the legend of Figure 1.

loser ecosystems had low diversities of plants and carnivorous species, but high herbivore diversity combined with small biomass.

Effect of evolutionary histories. To identify the conditions required for vulnerable ecosystems to develop, we conducted simulations of eco-fusion for three ecosystems with different evolutionary histories (types 2 to 4 ). The low primary production and the fluctuation of primary production did not clearly affect ecosystem vulnerability (Table S5). However, isolated evolution of an ecosystem strongly and significantly affected its vulnerability. Ecosystems with isolated evolution never won in all 500 simulations, whereas the control ecosystem was a winner in 442 of 500 simulations (Table S5).

The isolated ecosystems became so vulnerable because of the unique evolutionary processes they experienced. In isolated ecosystems, new lineages never appeared, so the only evolutionary pathway was diversification of a limited number of existing lineages through speciation (Table S6). In fact, species diversity within a lineage was much higher in the isolated ecosystems than in the winner ecosystems, at nearly 2.5 times the latter value (Table S6). Because species belonging to the same lineage had similar properties, they tended to become competitors. Therefore, plant species in isolated ecosystems suffered from more severe competition (Table S2, a higher number of competitors). As a result, the plant species diversity remained low, and the biomass of each plant species was also low (Table S2). Therefore, in isolated ecosystems, the same feedback loop as seen in loser ecosystems occurred, but it was stronger. As a result, the isolated ecosystems became extremely vulnerable. Indeed, many of the characteristics of loser ecosystems were also observed in the isolated ecosystems, but more conspicuously (Tables S1 and S2).

\section{Discussion}

Croft ${ }^{29}$ investigated the ratio of species diversity of carnivores to that of herbivores in mammal communities (both extant and fossil) from several continents. He found that the ratios in South America before
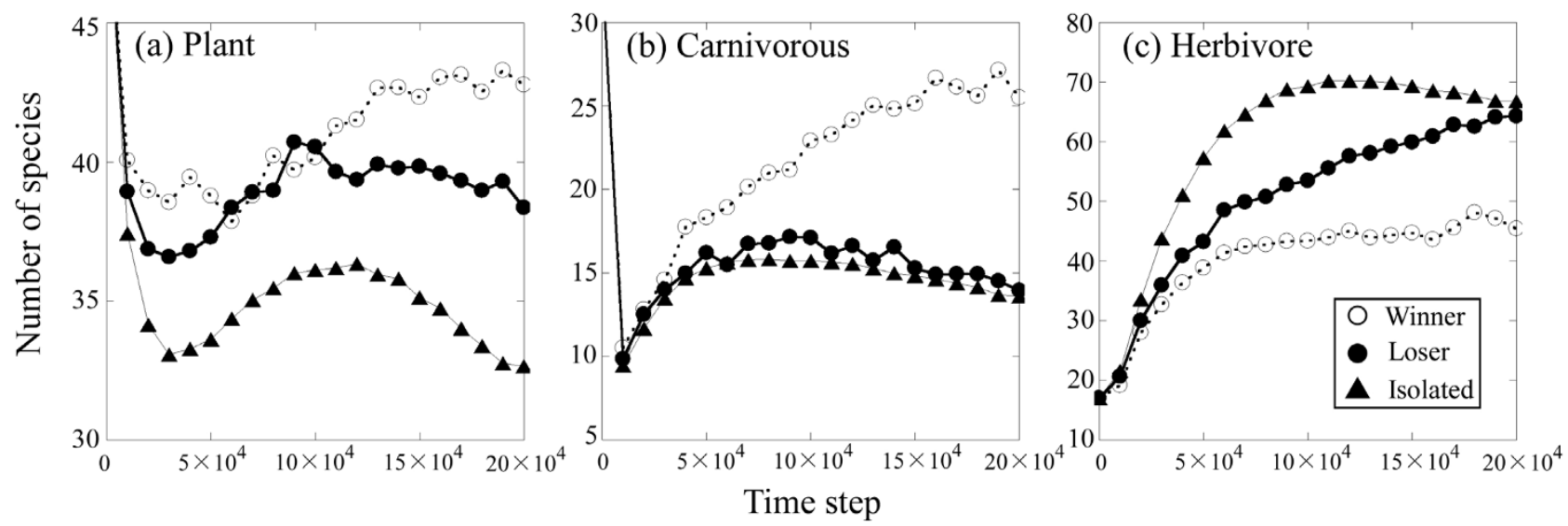

Figure $4 \mid$ Temporal changes in species diversity in winner, loser, and isolated ecosystems. Changes in diversity for (a) plants, (b) carnivorous species (carnivore and omnivore), and (c) herbivores in winner, loser, and isolated ecosystems. Data for winner and loser ecosystems are based on 294 simulations that exhibited an asymmetric extinction rate, and those for isolated ecosystems are based on 500 simulations. Data are shown at intervals of 10 000 time steps. 
the appearance of the Isthmus of Panama and in Australia were lower than those from the other continents; that is, these ecosystems exhibited the vulnerable structure described in the present study. When Croft incorporated terror birds as the top predators in South America before the appearance of the Isthmus of Panama, this did not affect the results ${ }^{29}$.

South America had been isolated from other continents for about 100 million years before the appearance of the Isthmus of Panama ${ }^{15}$. Australia has been isolated since the early Cenozoic. South American ecosystems were typically losers after the GABI, and Australian ecosystems have increasingly become losers as a result of biological invasions. On both continents, the processes described by our simulations may have proceeded while their ecosystems were isolated, leading to the development of vulnerability. Investigation of the changes in ecosystem structure on these continents may clarify the mechanisms responsible for this vulnerability. More clues about the mechanisms may be obtained if it becomes possible to define the ecosystem structure on the Indian Subcontinent before and after its collision with Eurasia, because the subcontinent would also have been isolated for a long time.

Comparing the results of this study with those of experimental work using micro- or mesocosms would deepen our understanding of eco-fusion. Experimental ecosystems should maintain high species diversity and should be constructed via evolutionary processes, as were the ecosystems in the current model. In this case, experiments with microbial microcosms may be preferable, because rapid evolution is frequently observed in these systems ${ }^{30,31}$. However, we should be cautious with research involving microbial ecosystems, which are very complicated and involve interspecific interactions that are not observed in ecosystems of large-bodied organisms ${ }^{32}$.

The model used in this study was not able to reproduce several patterns observed in real eco-fusions. Studies have shown that only a small proportion of the species pool of a donor ecosystem migrates to the recipient ecosystem. For example, only 2 to $11 \%$ of North American mammal genera and 2 to $7 \%$ of their South American counterparts took part in the $\mathrm{GABI}^{1}$. In the model, however, $43.5 \pm$ $12.3 \%$ (SD) of animal species and $76.6 \pm 7.01 \%$ of plant species migrated to the neighboring ecosystem. This discrepancy may be derived from the fact that only two ecosystems are considered in the model (the model in this study does not incorporate the physical distance between the locations). In the GABI, it may have been difficult for species living far from the Isthmus of Panama to take part in the biotic interchange, whereas, in the current model, all species were assumed to live near the connection point of the two ecosystems. Therefore, in future work, we should incorporate the concept of distance into the model (e.g., by increasing the number of ecosystems).

During the GABI, the direction of invasion was not simple. Savanna-adapted mammals and montane plants mainly migrated southward ${ }^{1,21}$, whereas rainforest species migrated in the opposite direction $^{1,33}$. The current model is too simple to reproduce such complex migration patterns. In order to properly simulate the direction of invasion, topography, climate, and species properties corresponding to these factors should be incorporated into the model.

The current model has a few additional limitations. Species evolution after eco-fusion is not considered, such that adaptive radiation after eco-fusion cannot be investigated. In the model, species are assumed to migrate simultaneously when the two ecosystems are united. In the case of the GABI, however, species migrated gradually as the Isthmus of Panama developed ${ }^{20}$ (waif dispersal $\rightarrow$ island hopping $\rightarrow$ walking). This simplification may affect the magnitude of the impact of species invasion. Therefore, in order to understand ecofusion more comprehensively, the model should be upgraded by incorporating these important factors.

1. Vermeij, G. J. When biotas meet: understanding biotic interchange. Science 253, 1099-1104 (1991).
2. Stehli, F. G. \& Webb, S. D. in Topics in Geobiology Vol. 4 (ed Stehli, F. G.) 532p (Springer, New York, 2012).

3. Por, F. D. Lessepsian migration: the influx of Red Sea biota into the Mediterranean by way of the Suez Canal. (Springer-Verlag, 1978).

4. Barash, A. \& Danin, D. Further additions to the knowledge of Indo-Pacific Mollusca in the Mediterranean Sea. Spixiana 9, 117-141 (1986).

5. Dana, T. F. Development of contemporary Eastern Pacific coral reefs. Mar. Biol. 33, 355-374 (1975)

6. Zinsmeister, W. J. \& Emerson, W. K. The role of passive dispersal in the distribution of hemipelagic invertebrate, with examples from the tropical Pacific Ocean. Veliger 22, 32-40 (1979).

7. Vermeij, G. J. Anatomy of an invasion: the trans-Arctic interchange. Paleobiology 17, 281-307 (1991)

8. Jablonski, D. \& Sepkoski, J. J. J. Paleobiology, community ecology, and scales of ecological pattern. Ecology 77, 1367-1378 (1996).

9. Webb, S. D. Ecogeography and the Great American Interchange. Paleobiology 17, 266-280 (1991).

10. Delsuc, F., Vizcaíno, S. F. \& Douzery, E. J. P. Influence of Tertiary paleoenvironmental changes on the diversification of South American mammals: a relaxed molecular clock study within xenarthrans. BMC Evol. Biol. 4, 11 (2004).

11. MacFadden, B. J. Extinct mammalian biodiversity of the ancient New World tropics. Trend. Ecol. Evol. 21, 157-165 (2006).

12. Eizenberg, J. F. \& Redford, K. H. in Mammalian Biology in South America (eds Mares, M. A. \& Genoways, H. H. ) 77-84 (Special Publication Series, vol. 6, Pymatuning Laboratory of Ecology, University of Pittsburgh, 1982).

13. Webb, S. D. in The Great American Biotic Interchange. Topics in Geobiology (eds Stehli, F. G. \& Webb, S. D.) (Springer, 2012).

14. Simpson, G. G. Holarctic mammalian faunas and continental relationships during the Cenozoic. Geol. Soc. Am. Bull. 58, 613-688 (1947).

15. Simpson, G. G. Splendid Isolation: The Curious History of South American Mammals. (Yale University Press, 1980).

16. Briggs, J. C. Dispersal of Tropical Marine Shore Animals: Coriolis Parameters or Competition? Nature 216, 350-350 (1967).

17. Moynihan, M. Successes and failures of tropical mammals and birds. Am. Nat. 105, 371-384 (1971).

18. Vermeij, G. J. Biogeography and Adaptation: Patterns of Marine Life. (Harvard University Press, 1978).

19. Marshall, L. G. in Biotic Crises in Ecological and Evolutionary Time (ed Nitecki, M. H.) (Academic Press, 1981).

20. Marshall, L. G. Land manmals and the Great American Interchange. Am. Sci. 76, 380-388 (1988).

21. Marshall, L. G., Webb, S. D., Sepkoski, J. J. J. \& Raup, D. M. Mammalian evolution and the Great American Interchange. Science 215, 1351-1357 (1982).

22. Repenning, C. A. Pleistocene mammalian faunas: Climate and evolution. Acta Zool. Fenn. 170, 173-176 (1985).

23. Lessa, E. P. \& Fariña, R. A. Reassessment of extinction patterns among the late Pleistocene mammals of South America. Palaeontology 39, 651-662 (1996)

24. Lindberg, D. R. in Paleobiology II (eds Briggs, D. G. E. \& Crowther, P. R.) 176-180 (Blackwell Science Ltd, 2001).

25. Gilpin, M. E. Community-level competition: asymmetrical dominance. Proc. Nat. Acad. Sci. USA 91, 3252-3254 (1994).

26. Yoshida, K. Evolutionary cause of the vulnerability of insular communities. Ecol. Modell. 210, 403-413 (2008).

27. Yoshida, K. The relationship between the duration of food web evolution and the vulnerability to biological invasion. Ecol. Complex. 5, 86-98 (2008).

28. Pimm, S. L. \& Lawton, J. H. The number of trophic levels in ecological communities. Nature 275, 542-544 (1977).

29. Croft, D. A. Do marsupials make good predators? Insight from predator-prey diversity ratios. Evol. Ecol. Res. 8, 1193-1214 (2006)

30. Fukami, T., Beaumont, H. J. E., Zhang, X. X. \& Rainey, P. B. Immigration history controls diversification in experimental adaptive radiation. Nature 446, 436-439 (2007).

31. Koeppel, A. F. et al. Speedy speciation in a bacterial microcosm: new species can arise as frequently as adaptations within a species. The ISME Journal 7, 1080-1091 (2013).

32. Faust, K. \& Raes, J. Microbial interactions: from networks to models. Nat. Rev. Microbiol. 10, 538-550 (2012).

33. Gentry, A. H. Neotropical floristic diversity: phytogeographical connections between Central and South America, Pleistocene climatic fluctuations, or an accident of the Andean orogeny? Annals Miss. Bot. Garden 69, 557-593 (1982).

\section{Acknowledgments}

We especially thank Kenji Takamura, Noriko Takamura, Akio Takenaka, Hiroya Yamano, and other members of Center for Environmental Biology and Ecosystem Studies, National Institute for Environmental studies for their enthusiastic guidance and discussion.

\section{Author contributions}

K.T. had the original idea of this study. K.Y. and K.T. designed the detail of this study. K.Y. conducted computer simulations, wrote the main manuscript text, and prepared all figures and supplementary information. K.Y. and K.T. approved the final manuscript. 


\section{Additional information}

Supplementary information accompanies this paper at http://www.nature.com/ scientificreports

Competing financial interests: The authors declare no competing financial interests. How to cite this article: Yoshida, K. \& Tokita, K. Properties of ecosystems that are vulnerable during eco-fusion. Sci. Rep. 5, 7939; DOI:10.1038/srep07939 (2015). (i) This work is licensed under a Creative Commons Attribution-NonCommercialNoDerivs 4.0 International License. The images or other third party material in this article are included in the article's Creative Commons license, unless indicated otherwise in the credit line; if the material is not included under the Creative Commons license, users will need to obtain permission from the license holder in order to reproduce the material. To view a copy of this license, visit http:// creativecommons.org/licenses/by-nc-nd/4.0/ 